\title{
Depletion-Induced Crystallization in Colloidal Rod-Sphere Mixtures
}

\author{
G. H. Koenderink, ${ }^{\dagger}$ G. A. Vliegenthart, ${ }^{\dagger}$ S. G. J . M. Kluijtmans, ${ }^{\dagger}$ \\ A. van Blaaderen, ${ }^{+} \neq$A. P. Philipse, ${ }^{\dagger}$ and H. N. W. Lekkerkerker*, ${ }^{\dagger}$ \\ Van't Hoff Laboratory for Physical and Colloid Chemistry, Debye Institute, Utrecht University, \\ Padualaan 8, $3584 \mathrm{CH}$ Utrecht, The Netherlands, and FOM Institute for Atomic and \\ Molecular Physics PB 41883, 1009 DB Amsterdam, The Netherlands
}

Received J anuary 14, 1999. In Final Form: April 30, 1999

\begin{abstract}
We report the observation of depletion-induced phase separation in a mixture of colloidal silica spheres and colloidal silica rods with light mi croscopy and confocal scanning laser microscopy. We show that very low rod concentrations are sufficient to induce sphere crystallization. This qualitatively agrees with theoretical predictions and demonstrates that rodlike colloidal particles are highly efficient depletion agents.
\end{abstract}

\section{Introduction}

Addition of small colloidal spheres to a dispersion of large colloidal spheres shifts the fluid-solid transition of this dispersion to lower volume fractions. ${ }^{1-7}$ This phenomenon is extensively studied theoretically ${ }^{8-11}$ and has also been confirmed by simulations. ${ }^{12-15}$ The phase transition is driven by so-called depletion interactions which can be explained in terms of purely repulsive interactions, between the small and large hard colloidal particles. ${ }^{16,17}$ Each large colloidal particle is surrounded by a shell, with a thickness of theradius of a small particle, into which the center of the small particle cannot penetrate. This excluded region is called the depletion zone. When two large particles approach each other such that their respective depletion zones start to overlap, the available volume for the small particles increases. This extra volume in turn causes the total entropy to increase and the free energy to decrease. In other words, the large particles experience an effective attraction. The corresponding "depletion potential" is to lowest order in the density given by ${ }^{16,17}$

* Author to whom all correspondence should be addressed. E-mail: h.n.w.lekkerkerker@chem.uu.nl.

† Utrecht University.

‡ FOM Institute for Atomic and Molecular Physics.

(1) van Duijneveldt, J . S.; Heinen, A. W.; Lekkerkerker, H. N. W. Europhys. Lett. 1993, 21, 369.

(2) Kaplan, P. D.; Rouke, J . L.; Yodh, A. G.; Pine, D. J . Phys. Rev. Lett. 1994, 72, 582.

(3) Steiner, U.; Meller, A.; Stavans, J . Phys. Rev. Lett. 1995, 74, 4750.

(4) Imhof, A.; Dhont, J. K. G. Phys. Rev. Lett. 1995, 75, 1662.

(5) Dinsmore, A. D.; Yodh, A. G.; Pine, D. J . Phys. Rev. E 1995, 52, 4045.

(6) Dinsmore, A. D.; Warren, P. B.; Poon, W. C. K.; Yodh, A. G. Europhys. Lett. 1997, 40, 337.

(7) Hobbie, E. K. Phys. Rev. Lett. 1998, 81, 3996.

(8) Lekkerkerker, H. N. W.; Stroobants, A. Physica A 1993, 195, 387.

(9) Rosenfeld, Y. Phys. Rev. Lett. 1994, 72, 3831.

(10) Poon, W. C. K.; Warren, P. B. Europhys. Lett. 1994, 28, 513.

(11) Gotzelmann, B.; Evans, R.; Dietrich, S. Phys. Rev. E. 1998, 57, 6785.

(12) Biben, T.; Bladon, P.; Frenkel, D. J . Phys.: Condens. Matter. 1996, 8, 10799.

(13) Dijkstra, M.; van Roij, R.; Evans, R. Phys. Rev. Lett. 1998, 80 3787.

(14) Dijkstra, M.; van Roij, R.; Evans, R. Phys. Rev. Lett. 1998, 81, 2268.

(15) Buhot, A.; Krauth, W. Phys. Rev. Lett. 1998, 80, 3787.

(16) Asakura, S.; Oosawa, F. J. Chem. Phys. 1954, 22, 1255.

(17) Mao, Y.; Cates, M. E.; Lekkerkerker, H. N. W. Physica A 1995, 222,10

$$
\mathrm{W}(\mathrm{h})=-3 \mathrm{k}_{\mathrm{B}} \mathrm{T} \phi_{\mathrm{s}} \frac{\mathrm{R}}{\sigma}\left(1-\frac{\mathrm{h}}{\sigma}\right)^{2}
$$

Here, $k_{B} T$ is the thermal energy, $\phi_{S}$ is the volume fraction of the small spheres, $\mathrm{R}$ is the radius of thelarge spheres, $\sigma$ is the diameter of thesmall spheres, and $h$ is the distance between the surfaces of the two large spheres. To obtain a rough estimate of the volume fraction needed to induce phase separation in a binary mixture of hard spheres, we assumethat such a phasetransition requires an attraction between the particles on the order of a few, say $3, k_{B} T$. This estimate is inspired by the work of Sperry, ${ }^{18}$ who found a minimum attraction of $2.7 \mathrm{k}_{\mathrm{B}} \mathrm{T}$ for the flocculation of latex spheres, and by simulation results ${ }^{19}$ for narrow potentials which give a value for the critical temperature of about 0.43 , that is, $\mathrm{k}_{\mathrm{B}} \mathrm{T}=2.3 \epsilon$, where $\epsilon$ is thewell depth. Equation 1 implies that, for example, for a size ratio $2 \mathrm{R} / \sigma$ $=10$ the depl etion interaction minimum equals $-3 \mathrm{k}_{\mathrm{B}} \mathrm{T}$ if the volume fraction of small spheres is about $20 \%$. It is precisely around these concentrations of small spheres that phase separation in the bulk is observed.1,3-5 I nterestingly, phase separation in the bulk is preceded by a fluid-solid phase separation at the wall.2,5,6 This is not entirely surprising, since the overlap volume of the depletion layers of a wall and a sphere is twice that for two spheres.

Although the depletion action of small hard particles is often discussed in terms of spheres, it is clear that particles with other shapes may al so inducea depletion interaction. This was already recognized by Asakura and Oosawa, ${ }^{20}$ who predicted that thin rodlike particles are especially effective for this purpose. In the limit that the length $L$ of the rodlike particles is much smaller than the radius $\mathrm{R}$ of the colloidal spheres, the depletion potential to the lowest order in density obeys ${ }^{20,21}$

$$
W(h)=-\frac{2}{3} k_{B} T \phi_{r} \frac{L}{D} \frac{R}{D}\left(1-\frac{h}{L}\right)^{3}
$$

In eq $2, \phi_{r}$ is the volume fraction of the rods and D is the

(18) Sperry, P. R. J . Colloid Interface Sci. 1984, 99, 97

(19) Vliegenthart, G. A.; Lekkerkerker, H. N. W. Physica A 1999 $263,378$.

(20) Asakura, S.; Oosawa, F. J . Polym. Sci. 1958, 32, 183.

(21) Mao, Y.; Cates, M. E.; Lekkerkerker, H. N. W. J. Chem. Phys. $1997,106,3721$ 

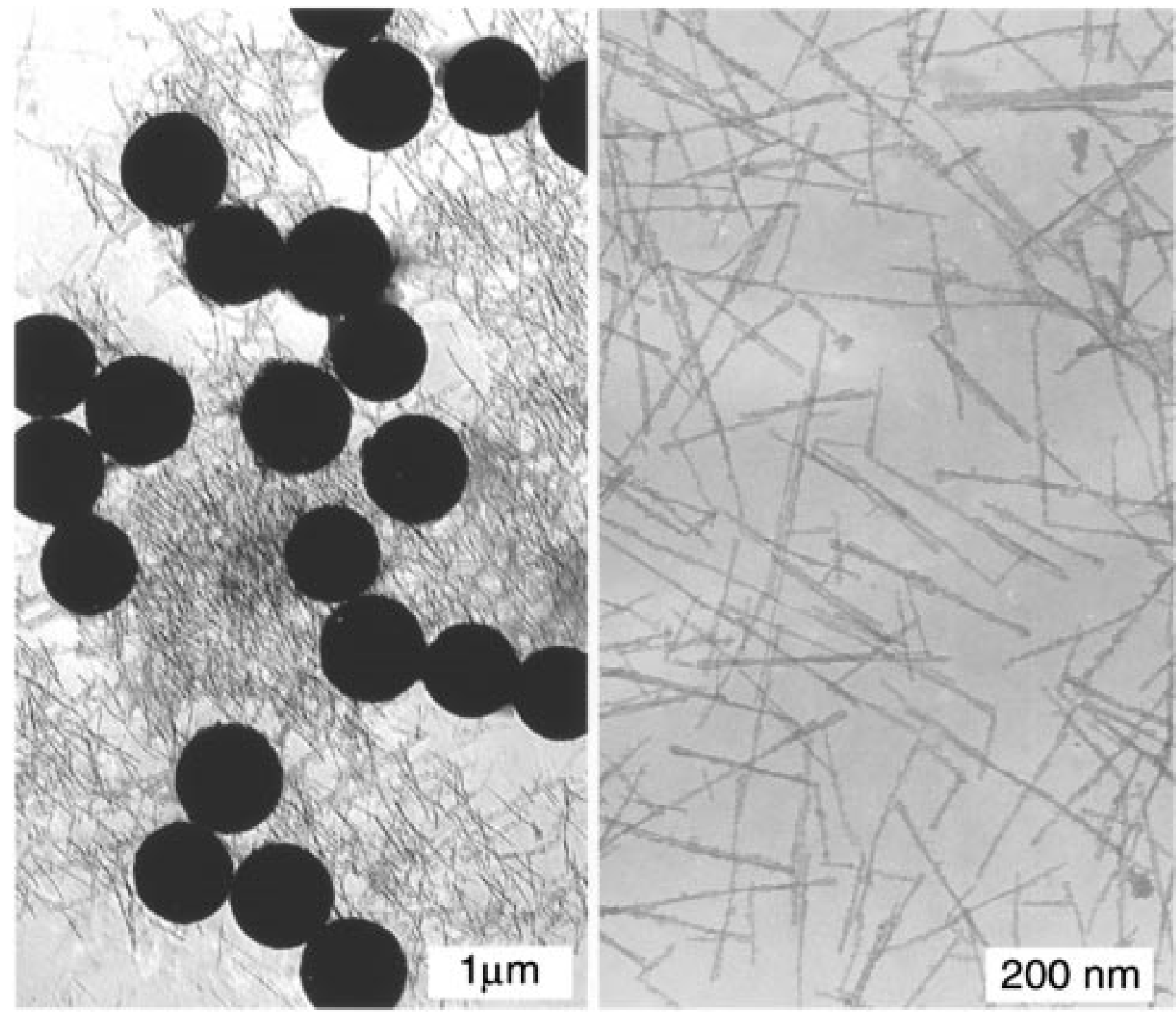

Figure 1. (A) Transmission electron micrograph of a mixture of silica spheres and silica-coated boehmite rods. (B) Electron micrograph of the rods.

diameter of the rodlike particles. From eq 2 we estimate that for the case $R / L=2$ and $L / D=20$ a contact value of the depletion interaction of $-3 \mathrm{k}_{\mathrm{B}} \mathrm{T}$ is obtained when the vol ume fraction $\phi_{\mathrm{r}}$ of rods is about $0.56 \%$. A moreaccurate calculation ${ }^{22}$ gives a slightly lower volumefraction of rods of $0.39 \%$ for which we expect demixing to occur. Clearly, rodlike particles are very efficient depletion agents; very low concentrations of rods are predicted to lead to phase separation. This also follows from detailed calculations on mixtures of spheres and infinitely thin rods ${ }^{23}$ or rods of a finite thickness. ${ }^{24}$

So far, no experimental evidence for the existence of a rod-sphere phase separation in dilute suspensions has been obtained, although some experiments on such mixtures have been reported. For instance, Tracy et al . ${ }^{25}$ studied the dynami cs of dispersions of the rodlikepolymer poly ( $\gamma$-benzyl- $\alpha$, L-glutamate) and silica spheres. They observed no phase separation, but this is likely duetothe

(22) Yaman, K.; J eppesen, C.; Marques, C. M. Europhys. Lett. 1998, $42,221$.

(23) Bolhuis, P.; Frenkel, D. J . Chem. Phys. 1994, 101, 9869.

(24) Vliegenthart, G. A.; Lekkerkerker, H. N. W. To be published. 1862 .
Iow concentrations of their samples. A related study was performed by Adams et al., ${ }^{26}$ who studied the phase behavior of rod-sphere mixtures of small spheres and large fd-virus rods. They found a number of new phases in addition to the well-known isotropic-nematic phase transition for purerod dispersions. Therod concentrations werewell abovetheisotropic-nematictransition density.

In this Letter, we present experimental evidence for a depletion-induced fluid-solid transition in a mixture of colloidal spheres and rigid colloidal rods at rod concentrations well below the isotropic-nematic transition. The transition is observed on the particle level (with microscopy) both in the bulk and near the wall.

\section{Experimental Section}

Our experimental system consists of colloidal silica spheres with a fluorescent core dispersed together with silica-coated boehmite rods in dimethylformamide (DMF) to which $0.001 \mathrm{M}$ $\mathrm{LiCl}$ has been added. In Figure 1 electron micrographs of our system are given. The silica spheres, labeled with fluorescein isothiocyanate(FITC), were prepared following the procedure of

(26) Adams, M.; Dogic, Z.; Keller, S. L.; Fraden, S. Nature 1998, 393, 

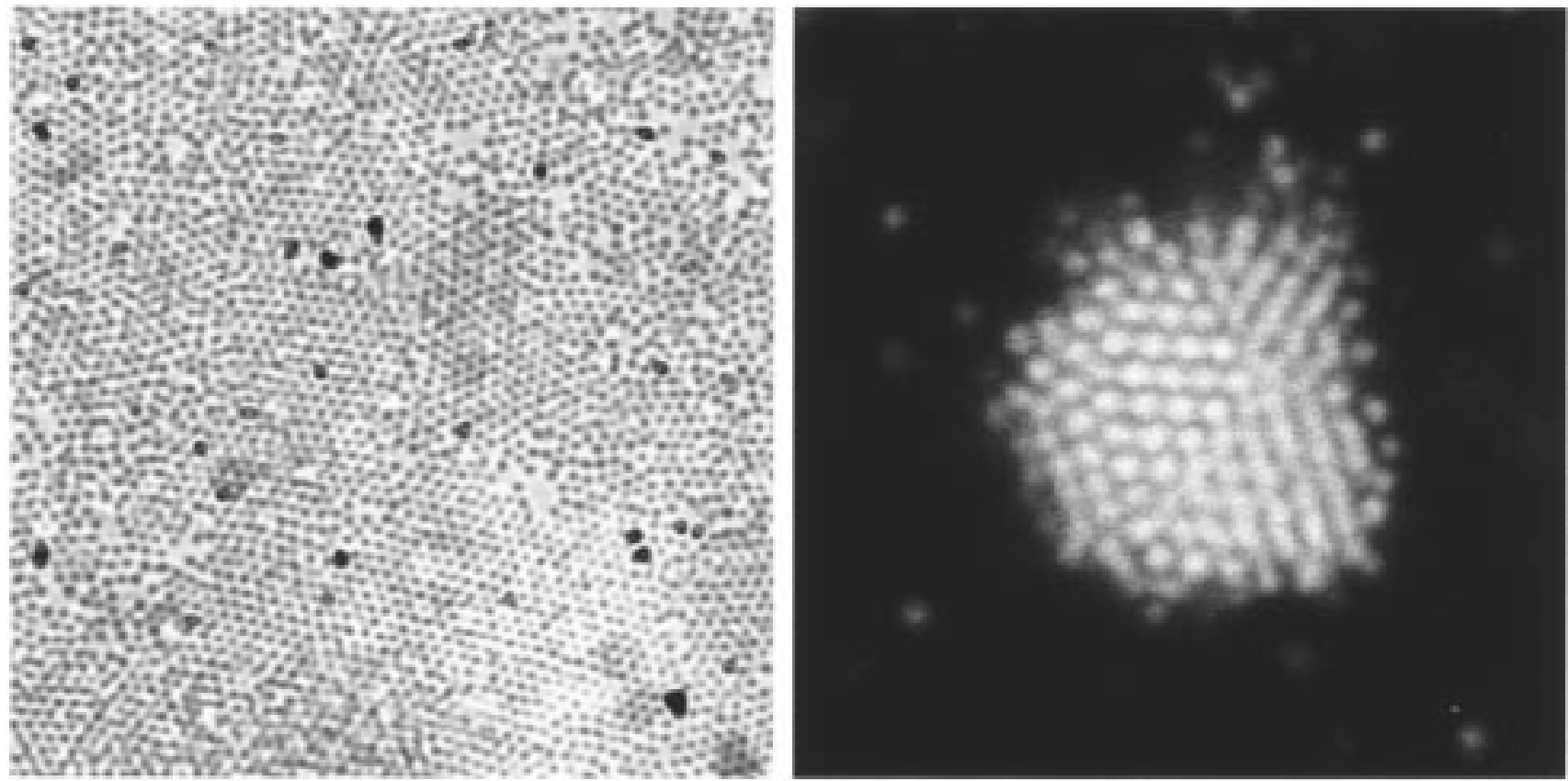

Figure 2. (A) Light microscopy picture of a wall crystallite in a system with a rod volume fraction of $0.33 \%$ and a sphere vol ume fraction of $1.0 \%$; the image size is $\pm 35 \times 35 \mu \mathrm{m}^{2}$. (B) Confocal microscopy image of a bulk crystallite in the same system; the image size is $\pm 17 \times 17 \mu \mathrm{m}^{2}$.

Van Blaaderen. ${ }^{27}$ The overall radius of the spheres is $370 \mathrm{~nm}$, and the radius of the fluorescent core is $186 \mathrm{~nm}$. Details on the synthesis and characterization of thespheres used in the present work can be found in ref 28. The FITC labeling of the particles, together with the large size of the particles, allows for direct observations by confocal microscopy. ${ }^{29,30}$ Thespheres form stable suspensions which sediment in a matter of hours due to their size. A stock solution of $19 \%$ volume fraction was prepared in DMF with $10 \mathrm{mM} \mathrm{LiCl}$.

Boehmite $(\gamma-\mathrm{AlOOH})$ rods of a length $\mathrm{L}=230 \pm 90 \mathrm{~nm}$ and a diameter $D=9 \pm 2 \mathrm{~nm}$ were synthesized according to the method of Buining. ${ }^{31}$ Since mixtures of bare silica spheres and bare boehmite rods immediately and irreversibly aggregate, ${ }^{32}$ the boehmiterods were coated with a thin layer of silica fol lowing Philipse et al. ${ }^{33}$ and Van Bruggen. ${ }^{34}$ The final stock solution contained $0.70 \%$ rods in pure DMF. This solution turned out to behighly viscous, which is common for charged rodl ikeparticles. ${ }^{35}$ The high viscosity may be partly due to residual attractive interactions between the rods. ${ }^{35}$

Mixtures of rods and spheres were prepared by weighing in the appropriate amounts of the stock dispersions and adjusting the salt concentration to $0.001 \mathrm{M}$ with $\mathrm{LiCl}$ in DMF. The $\mathrm{LiCl}$ is added toscreen doublelayer interactions between theparticles; theDebyelength for this $0.001 \mathrm{M} \mathrm{LiCl}$ dispersion is approximately $9 \mathrm{~nm}$. The silica rods and spheres disperse without aggregation upon mixing in DMF. Sedimentation measurements ${ }^{36}$ on the diluterod-spheremixtures werehighly reproducible, confirming that the mixtures are indeed stable.

Prior to measurements, a samplewith a particular composition was carefully mixed on a vortex mixer and a thin rectangular capillary ( $2 \mathrm{~mm}$ wide and $100 \mu \mathrm{m}$ thick, Vitrodynamics) was filled and sealed.

(27) van Blaaderen, A.; Vrij, A. Langmuir 1992, 8, 2921.

(28) Imhof, A.; Dhont, J. K. G. Phys. Rev. E. 1995, 52, 6344

(29) van Blaaderen, A.; Wiltzius, P. Science 1995, 270, 1177

(30) van Blaaderen, A.; Ruel, R.; Wiltzius, P. Nature 1997, 385, 321

(31) Buining, P. A.; Patmamanoharan, C.; J ansen, J . H. B.; Lekkerkerker, H. N. W. J Am. Ceram. Soc. 1991, 74, 1303.

(32) Philipse, A. P. Colloids Surf., A 1993, 80, 203.

(33) Philipse, A. P.; Nechifor, A. M.; Patmamanoharan, C. Langmuir 1994, 10, 4451.

(34) van Bruggen, M. P. B. Langmuir 1998, 14, 2245

(35) Wierenga, A. M.; Philipse, A. P. Colloids Surf. A 1998, 137, 355

(36) Kluijtmans, S. G. J . M.; Koenderink, G. H.; Philipse, A. P.; To be published.

\section{Results and Discussion}

In this Letter we will discuss two series of mixtures. In the first series the sphere volume fraction was fixed at $1.0 \%$ while varying the rod volume fraction between $0 \%$ and $0.70 \%$. In the second series, wefixed the spherevolume fraction at $2.5 \%$ while the rod volume fraction was varied between $0.25 \%$ and $0.60 \%$

In the first series the capillaries were stored in an upright position after sample preparation. In this series for rod volume fractions lower than $0.33 \%$, all spheres settled on the bottom of the cuvette in 1 day, leaving a sphere-free supernatant. However, in a sample with a rod volume fraction of $0.33 \%$, after 1 day, only part of the spheres had settled and at the same time we observed iridescent col ors resulting from $B$ ragg reflections over the whole length of the cuvette. A light microscopy (Zeiss Axioplan) study of the region cl osetothewall of thecuvette showed that thesamplehad becomeinhomogeneous. Parts of the sample showed liquid-like dynamics, as indicated by rapid Brownian movement of the individual particles and the absence of ordered structures. However, in other parts of the sample, crystallites appeared on the wall. In a matter of days these crystalline domains increased in size, and their internal structure became gradually more ordered. In Figure2A a typical microscopy imageis given of such a crystalline area at the glass wall, 1 week after sample preparation. This image clearly shows grain boundaries and defects. The sample was further investigated by confocal scanning laser microscopy (Leica TCS NT equipped with a krypton-argon laser, 1.4 NA 100× objective), which allows study of the samples more deeply into the bulk. With confocal microscopy some crystallites of about 1000 particles were observed. A typical example of such a bulk crystallite is shown in Figure 2B. The particles in the crystallites were somewhat mobile. The movement of the crystallite itself, and that of the single particles around it, is severely restricted due to the high solution viscosity, which also hinders their sedimentation. Samples with rod volume fractions of $0.70 \%$ became inhomogeneous within minutes. However, in contrast to 

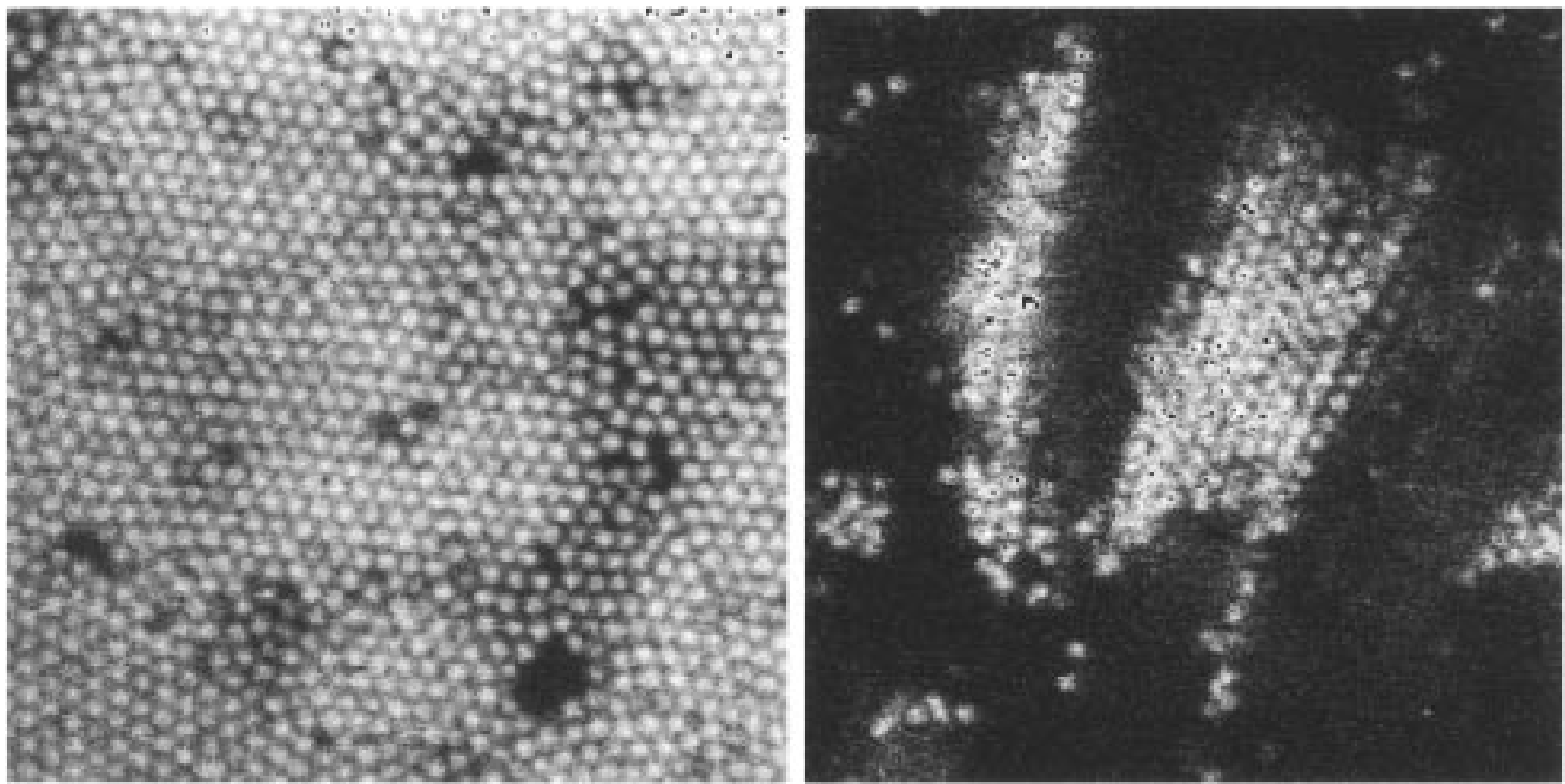

Figure 3. (A) Wall crystallites in a system with a rod volume fraction of $0.50 \%$ and a sphere volume fraction of $2.5 \%$. (B) Bulk crystallite in the same system, $30 \mu \mathrm{m}$ deep in the bulk. Both images were obtained by confocal microscopy; the image sizes are $50 \times 50 \mu \mathrm{m}^{2}$.

the case for the $0.33 \%$ sample, only small clusters of 5-15 particles were now seen with the light microscope and there were no crystals either at the wall or in the bulk.

In the second series of rod-sphere mixtures with the sphereconcentration fixed at $2.5 \%$, we madethefol lowing observations with the confocal microscope. For the rod volume fraction $0.25 \%$, no depletion effects by the rods wereobserved. The spheres sedimented on thelower glass wall within 15-30 min, forming a dense fluid layer (the capillaries in this case were kept in a horizontal position). When the rod volume fraction was increased to $0.33 \%$, a few small sphere clusters were observed in the bulk after 1 day. Spheres were immobilized near the upper glass wall, and small crystals had formed at the lower wall. Further increasing the rod volume fraction to $0.40 \%$ led to an increasing degree of crystallization at the wall and clustering in the bulk. The clustering and crystallization took place in a few hours. On going from $0.40 \%$ to $0.50 \%$ rods, clustering and subsequent crystallization both at the wall and in the bulk took only a few minutes. Figure $3 A$ shows small crystals which appeared at thewall in the system containing $0.50 \%$ rods. Compared to the system of Figure 2A, where the rod and sphere volume fraction were lower, these crystals were formed much faster. In Figure 3B a large bulk crystal from this same system is shown. Compared to the system of Figure 2B, the bulk crystal is larger in size (10 000 particles).

We concludefrom these findings that phase separation in rod-sphere mixtures can occur at very low concentrati ons of rods and spheres. An increase both of the sphere concentration and of the rod concentration lead to an increase of the crystallization rate. The optimum conditions for crystallization in our system are found to lie in a narrow window of rod volume fractions from $0.33 \%$ to $0.50 \%$. At rod concentrations bel ow $\phi_{\mathrm{r}}=0.33 \%$, thespheres rapidly sediment without undergoing any depletioninduced fluid-solid phase transition. On the other hand, at high rod volume fractions $\phi_{r}>0.60 \%$, the depletion action must bestrong but, neverthel ess, again nodepletion effects are observed. In this case this is dueto an extreme increase in the viscosity, ${ }^{36}$ which almost completely immobilizes thespheres within a few minutes after sample preparation.

Thelower end of the concentration rangeat which phase separation is found seems to be in agreement with the theoretical estimate given in the Introduction and with theoretical phase diagrams of sphere-rod mixtures presented in refs 23 and 24 . This strongly suggests that wehaveindeed observed entropy-driven fluid-solid phase separation. The low rod concentration needed to induce the phase separation also confirms the prediction of Asakura and Oosawa ${ }^{20}$ that rods are much more efficient depletion agentsthan small spheres. Thefairly rapid phase separation combined with the possibility of directly visualizing individual spheres with CSLM makes our rodspheredispersion promising for detailed studies of colloidal crystallization pathways. If the size of the spheres is increased somewhat, this will allow for quantitative determination of their positions. ${ }^{30}$

Acknowledgment. Wethank Michel van Bruggen for sharing his expertise on the boehmiterods, Arnout I mhof for providing us with thefluorescent silica spheres, Willem Kegel for his help with thelight microscopy experiments, and Paul van der Schoot for a critical reading of the manuscript. This work was supported by the Stichting voor Fundamenteel Onderzoek der Materie (F oundation for Fundamental Research on Matter), which is part of the Nederlandse Organisatie voor Wetenschappelijk Onderzoek (Netherlands Organization for the Advancement of Research).

\section{LA990038T}

